\section{THE TALE OF SIR2}

The Sir2 family of $\mathrm{NAD}^{+}$-dependent deacetylases, or sirtuins, regulate a wide variety of cellular processes, including gene silencing, and are involved in determining lifespan. Conserved from bacteria to humans, sirtuins operate by a mechanism in which $\mathrm{NAD}^{+}$is consumed and the putative second messenger 0 -acetyl-ADP-ribose is produced. Recently, a sirtuin in a

Salmonella strain was shown to deacetylate and thereby activate acetyl-CoA synthetase (AceCS), the enzyme that converts acetate to the usable metabolite acetyl-coenzyme A. Hallows et al. now find that mammalian cells also regulate AceCS through reversible acetylation, and that sirtuins have an important role in activation of specific AceCS subtypes. In mammals, there are two known AceCSs: cytoplasmic AceCS1 and mitochondrial AceCS2. The authors found that bacterial protein acetyltransferase (PAT) was capable of acetylating AceCS1 in vitro. When AceCS1 was coexpressed in mammalian cells with the seven human sirtuin homologues, only SIRT1 markedly reduced endogenous AceCS1 acetylation (by an as-yet-unidentified mammalian PAT). In a radioassay, AceCS1 productivity was virtually abolished by acetylation but was rescued by SIRT1. Parallel work demonstrated that SIRT3-confined to mitochondria, along with AceCS2 - was responsible for AceCS2 deacetylation, confirming the notion that acetylation is an on-off switch for AceCS. Further work to determine how sirtuins are regulated may illuminate the connection between aging and metabolism. (Proc. Natl. Acad. Sci. USA 103, 10230-10235, 2006)

\section{A natural tryptophan zipper}

Aromatic stacking has long been

known to be a biologically rel-

evant noncovalent interaction;

the DNA double helix represents the

classic example. Yet observation of a

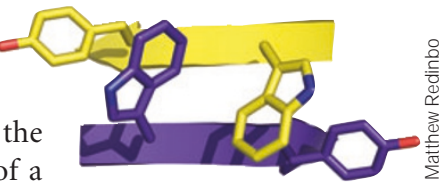

corresponding array of $\pi-\pi$ interac-

tions in proteinaceous systems has been limited to the design com-

munity. Noble et al. now report the first example of a naturally encoded stacking motif in the human pregnane $\mathrm{X}$ receptor (PXR), a member of the nuclear receptor superfamily. PXRs are unique in this family owing to the insertion of an extra 50-residue sequence within an otherwise well-conserved fold. Two residues of this sequence, a tryptophan and a tyrosine, have been identified as key mediators of the formation of a functional homodimer. Although this dimer has been observed in crystal structures before, this report is the first to demonstrate that it is biologically relevant. Mutation of the identified residues prevented association completely in vitro and diminished the response to ligand in vivo. This altered behavior was identified as being due to a defect in coactivator (SRC-1) recruitment, rather than to changes in ligand or DNA binding or in the ability of PXR to interact with its heterodimer partner RXR $\alpha$. The structure of the tyrosine-tryptophan dimer is in good agreement with that of a previously designed tryptophan zipper (Trp-Zip) which is known to provide outstanding stability. The observation of a functional role for this interaction provides new impetus for investigations of this structural motif. (Biochemistry, published online 22 June 2006, doi: 10.1021/bi0602821)

CG

Research Highlights written by Mirella Bucci, Catherine Goodman, Kaspar Mossman and Terry L. Sheppard

\section{Illuminating cellular RNA}

The selective staining of cells and organelles by organic dyes is an important technique in cellular studies. For example, DNA-binding dyes such as DAPI or Hoechst 33258 have been invaluable for visualizing chromosomes. More recently, modern microscopy and selective fluorescence labeling techniques have made it possible to obtain unparalleled views of living cells in real time. Although commercially available fluorescent dyes exist for most biomolecules or cellular compartments, the selective staining of RNA has met with limited success. Now Li et al. have constructed an expanded chemical library of dyes based on heterocyclic styrene chromophores and screened it for molecules that bind RNA in vitro and localize to the nucleoli of live cells. Eighty-eight compounds identified by the screen were synthesized on large scale, and a secondary imaging strategy across multiple cell types was used to identify three promising dye candidates. These compounds all contained a cationic quinaldine moiety, had optimal working concentrations in the low micromolar range, and showed limited toxicity and photobleaching under standard imaging conditions. The authors demonstrated that these styryl dyes bound selectively to RNA over DNA and could be used along with DNA-targeting dyes such as DAPI to label DNA and RNA simultaneously in the same cell. Although future studies will be required to reveal the structural basis of selective RNA binding, these compounds offer considerable promise in comparison to currently available methods for visualizing RNA in cells. (Chem. Biol. 13, 615-623, 2006)

\section{Avoiding trouble with Tribbles}

Mice with an insulin receptor (IR) mutation in adipose tissue are lean and live longer than normal mice, perhaps as a result of enhanced fatty acid oxidation (FAO). The expression of a 'pseudokinase,' Tribbles 3 (TRB3), is increased during fasting and when IR signaling is lost. TRB3, catalytically inactive, is known to have a role in enhancing the ubiquitin ligase machinery and therefore protein degradation. Qi et al. now show that TRB3 is an adaptor protein that links the degradative machinery to lipid metabolism. The authors expressed TRB3 in adipose tissue in mice. Even though they consumed more food than wild-type mice and had similar physical activity, these transgenic mice gained weight more slowly. The size of the average adipocyte in TRB3 mice was lower, their $\mathrm{O}_{2}$ consumption was increased, and their core body temperature was higher. Further, TRB3 mice had increased rates of FAO and increased expression of FAO enzymes. The authors found that, under fasting conditions, TRB3 interacts with and inhibits ACC1, the rate-limiting enzyme in fatty acid synthesis. Simultaneously, TRB3 interacted with COP1, a ubiquitin ligase; this interaction was necessary for ubiquitination and ultimate destruction of ACC1. So, TRB3 stimulates FAO in adipose tissue during fasting through COP1-mediated degradation of ACC1. TRB3 represents a pathway, parallel to others known to reguthat is turned on during fasting to limit weight gain and insulin resistance. (Science 312, 1763-1766, 2006)

$M B$ 